\title{
The Minimum Latency Problem is NP-hard for Weighted Trees
}

\author{
René Sitters \\ r.a.sitters@tue.nl \\ Department of Mathematics and Computer Science \\ Technische Universiteit Eindhoven \\ P.O.Box 513, 5600 MB Eindhoven, The Netherlands
}

\begin{abstract}
In the minimum latency problem (MLP) we are given $n$ points $v_{1}, \ldots, v_{n}$ and a distance $d\left(v_{i}, v_{j}\right)$ between any pair of points. We have to find a tour, starting at $v_{1}$ and visiting all points, for which the sum of arrival times is minimal. The arrival time at a point $v_{i}$ is the traveled distance from $v_{1}$ to $v_{i}$ in the tour. The minimum latency problem is MAX-SNP-hard for general metric spaces, but the complexity for the problem where the metric is given by an edge-weighted tree has been a long-standing open problem. We show that the minimum latency problem is NP-hard for trees even with weights in $\{0,1\}$.
\end{abstract}

\section{Introduction}

Given $n$ points $v_{1}, \ldots, v_{n}$ and a distance between any pair of points, the minimum latency problem asks for a tour $\pi$, starting at $v_{1}$ and visiting all points, for which the sum of the arrival times $d_{\pi}\left(v_{1}, v_{i}\right)$ is minimum, where the arrival time is defined as the traveled distance from $v_{1}$ to $v_{i}$ in tour $\pi$. The minimum latency problem has been well-studied in operations research, where it is also known as the traveling repairman problem and the delivery man problem. Unlike the traveling salesman problem, where the objective is minimizing maximum arrival time and therefore is server oriented, the MLP is client oriented. Interesting applications of the MLP are diskhead scheduling and searching information in a network [4],[12] (for example in the world wide web), where the MLP can be used to minimize expected search time.

The MLP was proven to be NP-complete for general metric spaces by Sahni and Gonzalez [16]. In fact both the traveling salesman problem and the MLP are MAX-SNP-hard for general metric spaces, but the minimum latency problem has a reputation for being much harder than the traveling salesman problem. The first constant-factor approximation algorithm for general metric spaces was a 144-approximation algorithm given by Blum et al. [6]. A 7.18-approximation algorithm follows from a result of Goemans and Kleinberg [11] and an improved version of Garg's algorithm [10] for the $k$-MST problem. The improvement is mentioned in the papers by Arora and Karakostas [3] and Chudak et al. [7]. We 
refer to the papers of Goemans and Kleinberg [11], Arora and Karakostas [2], Ausiello et al. [4] and Lenstra et al. [13] for a recent overview of the MLP and related problems.

\section{The minimum latency problem for trees}

The problems in which the metric is defined by points on the line or an edgeweighted tree are denoted by the line- $M L P$ and the tree-MLP respectively. The line-MLP can be solved in $\mathrm{O}\left(n^{2}\right)$ time by dynamic programming, as was shown by Afrati et al. [1]. Recently a linear time algorithm has been proposed by García et al. [8]. Notice that the traveling salesman problem is trivial even for edgeweighted trees. However, for the tree-MLP no exact polynomial time algorithm is known. Classifying the complexity of the MLP for edge-weighted trees has been mentioned as an open problem in many papers [3-6, 11, 12, 15, 19, 20]. For example Goemans and Kleinberg [11] write that 'the MLP is not known to be NP-hard in weighted trees, so it is worth considering whether it could be solved optimally'. The first attempt to solve the tree-MLP was made by Minieka [14] who gave an $O\left(n^{k+1}\right)$ algorithm, where $n$ is the number of vertices and $k$ is the number of leaves. Koutsoupias et al. mention that the dynamic programming solution for the line-MLP can be extended to a $O\left(n^{k}\right)$ dynamic programming algorithm for the tree-MLP. Blum et al. [6] show that dynamic programming gives an $O\left(n^{2}\right)$ algorithm for the tree-MLP on trees with diameter at most 3. $\mathrm{Wu}[20]$ puts the dynamic programming approach for the MLP in a more general framework. If the tree is unweighted, then a tour is optimal if and only if it is a depth-first search. Proofs have been given by several authors [6], [15].

Goemans and Kleinberg [11] gave a 3.59-approximation algorithm for the tree-MLP, improving the 8-approximation algorithm given by Blum et al. [6]. Arora and Karakostas [2] give a quasipolynomial-time approximation scheme for weighted trees and for Euclidean spaces with fixed dimension. The performance of depth-first search algorithms has been studied by Webb [19].

\section{$3 \quad$ Implications and open problems}

A problem almost equivalent to the MLP is the graph searching problem (GSP), in which an arbitrary probability distribution on the nodes is given and we want to minimize the sum of all arrival times multiplied by their probability. If we imagine that the probabilities represent a probability that an object is hidden at the vertex, then the optimal tour minimizes the expected search time. From Theorem 1 it follows immediately that the GSP is strongly NP-hard for edgeweighted trees. Moreover, Corollary 1 implies that the GSP is NP-hard for trees with unit edge-lengths.

Another interesting problem is finding the search ratio of a graph, introduced by Koutsoupias et al. [12]. Again one has to find an object hidden in a vertex not known to the server, but instead of a distribution on the vertices one is facing an adversary who chooses at which vertex it hides the object. The search ratio is 
then defined as the optimal competitive ratio, where the competitive ratio is the distance traveled by the server divided by the length of the shortest path from the origin to the object. Koutsoupias et al. [12] show that both the randomized and the deterministic version are MAX-SNP-hard for general graphs. They note that computing the (randomized) search ratio for trees is a 'surprisingly tough problem' but show that, if one can solve the GSP for a class of graphs in polynomial time, then by duality one can solve the randomized search ratio problem for that same class of graphs. Our result excludes this tool for finding a polynomial time algorithm for the randomized search ratio problem for trees. However, polynomial time approximation algorithms for the MLP may be transferable to the randomized search ratio problem [12].

The MLP is NP-hard for the metric with distances 1 and 2, but the complexity for trees with edge distance 1 and 2 does not follow from our result. We conjecture that this problem is polynomially solvable.

The 3.59-approximation algorithm of Goemans and Kleinberg gives the current best ratio for the tree-MLP. However this algorithm hardly uses characteristics of the tree-metric. We conjecture that a significant improvement of this ratio is possible.

The value of the optimal MLP-tour clearly depends on the given starting vertex. To find the best starting vertex we could repeatedly run an algorithm that solves the MLP, each time using a different starting vertex. However, SichmiLevi and Berman [17] notice that for general metric spaces one run suffices. Wu [20] gives an $\mathrm{O}\left(n^{2}\right)$ algorithm for this problem on the line, and Minieka [15] notices that for unweighted trees any vertex at the end of a longest path can be taken as the best starting vertex. It is unknown to us whether, for weighted trees and for general metric spaces, there is a more subtle way than solving an MLP instance to find the best starting vertex.

Koutsoupias et al. [12] mention that the MLP is conjectured to be NP-hard even for caterpillars (a path with edges sticking out). This conjecture remains unresolved.

In the forthcoming paper by Lenstra et al. [13] the authors define a large class of so called dial-a-ride problems. In this more general framework servers have to transport items from a source to a destination in a metric space. The class contains about 8,000 problems, from which all but 72 have been classified as NP-hard or solvable in polynomial time ${ }^{1}$. As one of the most interesting open problems, the authors mention the minimum latency problem with release dates and where the metric is the real line. This problem was already mentioned as an interesting open problem by Tsitsiklis [18].

\section{Proof of NP-hardness}

We define an instance of the tree-MLP as a tree with root $r$ and weights on the edges and the vertices. Notice that we can polynomially reduce any such instance

\footnotetext{
${ }^{1}$ By February 2002
} 
to an instance with unit vertex-weights by replacing a vertex with weight $w$ by $w$ vertices at distance zero from one another. Of course this only applies if all vertex-weights are polynomially bounded. For clarity we shall use the terms edge length and vertex weight. We will use the term total completion time for the sum of the weighted completion times. We assume that the weight of the starting vertex is zero and that the server always ends its tour in the origin.

To facilitate the exposition we assume that the server travels at unit speed such that distance traveled and elapsed time are equal. Given an instance of the MLP on a tree, let $T$ be an optimal tour and let $t_{0}=0, t_{1}, \ldots, t_{k}$ be the moments at which the server is in the origin. Notice that $t_{k}$ is equal to the length of $T$ since we assumed that the server ends in the origin. Let $T_{i}$ be the subtour between time $t_{i-1}$ and $t_{i}, 1 \leq i \leq k$, and let $\left|T_{i}\right|$ resp. $W_{i}$ be the length resp. total weight of this subtour. Then the we have the following lemma.

Lemma 1. For any optimal tour $T$ the following holds.

(i) $\frac{W_{1}}{\left|T_{1}\right|} \geq \frac{W_{2}}{\left|T_{2}\right|} \geq \ldots \geq \frac{W_{k}}{\left|T_{k}\right|}$.

(ii) If $\frac{W_{i}}{\left|T_{i}\right|}=\frac{W_{j}}{\left|T_{j}\right|}$ for some $i, j \in\{1, \ldots, k\}$, then the total completion time remains the same if the subtours $T_{i}$ and $T_{j}$ swap their position on $T$.

Proof. (i) We use a simple interchange argument. Assume that for some $i \frac{W_{i}}{\left|T_{i}\right|}<$ $\frac{W_{i+1}}{\left|T_{i+1}\right|}$. If we change the order of the subtours $T_{i}$ and $T_{i+1}$, then the increase in the total completion time is $W_{i}\left|T_{i+1}\right|-W_{i+1}\left|T_{i}\right|<0$. Now (ii) follows directly from the proof of (i).

To prove NP-hardness for the tree-MLP we give a reduction from the 3-Partition problem, which was proven to be NP-hard in the strong sense by Garey and Johnson [9].

\section{3-Partition}

Instance: A multiset of natural numbers $A=\left\{p_{1}, p_{2} \ldots, p_{3 n}\right\}$, with $P / 4<p_{i}<$ $P / 2$ for all $i \in\{1, \ldots, 3 n\}$, and a number $\mathrm{P}$ such that $p_{1}+\cdots+p_{3 n}=n P$.

Question: Is it possible to partition the set $A$ in $n$ sets $A_{1}, \ldots, A_{n}$ such that $\sum_{p_{i} \in A_{j}} p_{i}=P$ for all $j \in\{1, \ldots, n\} ?$

Theorem 1. The minimum latency problem is strongly NP-hard for weighted trees.

Proof. Given an instance of 3-Partition with the notation as described above, we define $a_{i}=K p_{i}$ for all $i \in\{1, \ldots, 3 n\}$ and $Q=K P$, where $K=2 P n^{4}$. We define a tree on $3 n(n+2)+1$ vertices as follows.

For each $i \in\{1, \ldots, 3 n\}$ we construct a path $\left(r, v_{i 1}, v_{i 2}, \ldots, v_{i n}, z_{i}\right)$. All these paths start in the root of the tree, which is appointed as the origin of the MLPinstance. To each of these paths an extra vertex $u_{i}$ is attached through the edge $\left(v_{i 1}, u_{i}\right)$ (see Figure 1). For the definition of the lengths of the edges in this tree 
we introduce the numbers $m$ and $l_{i}, i \in 1, \ldots, 3 n$, and choose their value appropriately later. The lengths of the edges are:

$$
\begin{array}{ll}
d\left(r, v_{i 1}\right)=l_{i}, & i=1, \ldots, 3 n \\
d\left(v_{i j}, v_{i, j+1}\right)=a_{i}, & i=1, \ldots, 3 n, j=1, \ldots, n-1 ; \\
d\left(v_{i 1}, u_{i}\right)=2 Q a_{i}, & i=1, \ldots, 3 n \\
d\left(v_{i n}, z_{i}\right)=m, & i=1, \ldots, 3 n .
\end{array}
$$

The weights on the vertices are:

$$
\begin{array}{ll}
w\left(v_{i 1}\right)=a_{i}, & i=1, \ldots, 3 n ; \\
w\left(v_{i j}\right)=n-j+1, & i=1, \ldots, 3 n, j=2, \ldots, n ; \\
w\left(z_{i}\right)=a_{i}, & i=1, \ldots, 3 n ; \\
w\left(u_{i}\right)=1, & i=1, \ldots, 3 n .
\end{array}
$$

We call the subtree rooted at $r$ and constituted by the path $\left(r, v_{i 1}, \ldots, v_{i n}, z_{i}\right)$ and the edge $\left(v_{i 1}, u_{i}\right)$ the root-branch $i, i=1, \ldots, 3 n$.

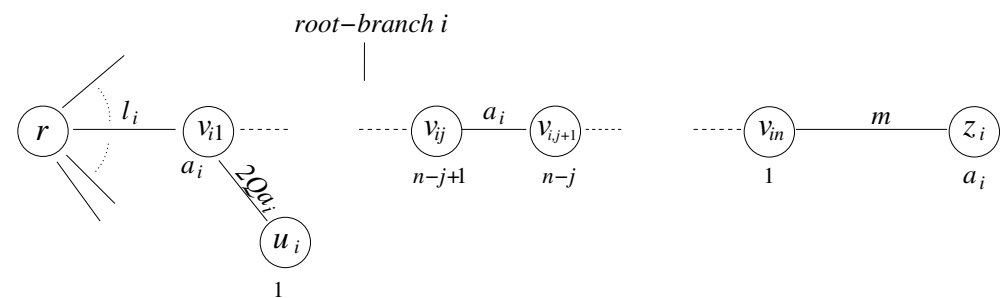

Fig. 1. Sketch of the MLP instance.

If the values $m$ and $l_{i}$ are chosen appropriately large it is easy to see that an optimal tour satisfies the following properties for all $i$ in $\{1, \ldots, 3 n\}$ :

(a) each edge $\left(r, v_{i 1}\right)$ is traversed exactly once in each direction;

(b) vertex $u_{i}$ is visited before vertex $z_{i}$.

Moreover, we can choose $l_{i}, i=1, \ldots, 3 n$, such that for all $i$ and $h$ in $\{1, \ldots, 3 n\}$

(c) $\frac{W_{i}}{L_{i}}=\frac{W_{h}}{L_{h}}$, where $W_{i}$ and $L_{i}$ are, respectively, the sum of all vertex-weights and the sum of all edge-lengths of root-branch $i$.

With respect to (c) we note that choosing $l_{i}=M\left(2 a_{i}+n(n-1) / 2+1\right)-(2 Q+$ $n-1) a_{i}-m$ yields $\frac{W_{i}}{L_{i}}=M$, for all $i \in\{1, \ldots, 3 n\}$, where $M$ is a large number. It is intuitively clear that we can bound the numbers $m$ and $M$ by a polynomial in the size of the 3-Partition instance. A proof for this is submitted at the end of this proof.

By (a) and (b) there are only $n$ different ways for the server to traverse rootbranch $i$ : for $k=2, \ldots, n$, we call the subtour $\left(r, v_{i 1}, \ldots, v_{i k}, v_{i, k-1}, \ldots, v_{i 1}, u_{i}\right.$, $\left.v_{i 1}, \ldots, v_{i n}, z_{i}, v_{i n}, \ldots, v_{i 1}, r\right)$ the $k$-tour. The tour $\left(r, v_{i 1}, u_{i}, v_{i 1}, \ldots, v_{i n}, z_{i}\right.$, 
$\left.v_{i n}, \ldots, v_{i 1}, r\right)$ is a 1 -tour. Consider an optimal tour. Renumber the root branches according to the $k$-tour by which they are traversed: root branches $1, \ldots, i_{1}$ are traversed by a 1 -tour, $i_{1}+1, \ldots, i_{2}$ by a 2 -tour, etc. until $i_{n-1}+1, \ldots, i_{n}=3 n$ being the root branches traversed by an $n$-tour. We know from Lemma 1(i) and (c) that in an optimal tour all root-branches $1, \ldots, i_{1}$ are served first followed by the root branches $i_{1}+1, \ldots, i_{2}$, etc.

Now consider the tour in which all root-branches are traversed by a 1-tour. We will compare the optimal tour with this tour. Due to Lemma 1(ii) and (c) we may assume that the order in which the root-branches are served is the same for both tours.

If root-branch $i$ is served by a $k$-tour, then compared to serving this root-branch by a 1 -tour, this will reduce the completion time of all vertices $v_{i j}$ by $2 \leq j \leq k$ by $4 Q a_{i}$ and increase the completion time of all vertices $v_{i j}$ by $k+1 \leq j \leq n$ and vertices $u_{i}$ and $z_{i}$ by $2(k-1) a_{i}$. It also increases the completion time of all vertices in root-branches $h$ for $h \geq i+1$ by $2(k-1) a_{i}$.

Thus the total decrease of serving according to the optimal solution instead of the all 1-tour solution is

$$
\sum_{k=2}^{n} \sum_{i=i_{k-1}+1}^{3 n} 4(n-k+1) Q a_{i}=\sum_{k=2}^{n}\left(4(n-k+1) Q \sum_{i=i_{k-1}+1}^{3 n} a_{i}\right) .
$$

Let us now first study the total increase $R$ due to a delay of all vertices except the vertices $z_{i}$ and $v_{i 1}, i=1, \ldots, 3 n$. There are $3 n^{2}$ of them and their total weight is $3 n\left(\frac{1}{2} n(n-1)+1\right)<2 n^{3}$. The delay for each of them is at most $2(n-1) Q$. Therefore, $R<4 Q n^{4}$.

To study the total increase due to a delay of the vertices $v_{i 1}$ and $z_{i}, i=$ $1, \ldots, 3 n$, consider a root branch $h$ that is served by a $k$-tour, i.e., $i_{k} \leq h<i_{k+1}$. Compared to serving it by a 1 -tour, the vertex $v_{h 1}$ is not delayed, but the vertex $z_{h}$ is delayed by an amount $2(k-1) a_{h}$, as noted before, which multiplied by its weight gives an increase in objective value of $2(k-1) a_{h}^{2}$. Moreover, all vertices $v_{i 1}$ and $z_{i}$ for $i>h$ receive a delay of $2(k-1) a_{h}$ by this $k$-tour. Thus, the total increase by this $k$-tour due to delay of vertices $v_{i 1}$ and $z_{i}, i=h, \ldots, 3 n$ amounts to

$$
2(k-1) a_{h}\left(a_{h}+\sum_{i=h+1}^{3 n} 2 a_{i}\right)
$$

The total increase of the optimal solution over the all 1-tour solution is then given by

$$
R+\sum_{k=2}^{n} \sum_{h=i_{k-1}+1}^{i_{k}} 2(k-1) a_{h}\left(a_{h}+\sum_{i=h+1}^{3 n} 2 a_{i}\right)=R+\sum_{k=2}^{n} 2\left(\sum_{i=i_{k-1}+1}^{3 n} a_{i}\right)^{2} .
$$


Let $C^{O P T}$ and $C$ be the total completion times, respectively, for the optimal tour and the all 1-tour solution. Combining (1) and (2) we obtain

$$
C^{O P T}=C+R+\sum_{k=2}^{n}\left(2\left(\sum_{i=i_{k-1}+1}^{3 n} a_{i}\right)^{2}-4 Q(n-k+1) \sum_{i=i_{k-1}+1}^{3 n} a_{i}\right) .
$$

For each $k \in\{2, \ldots, n\}$, writing $b_{k}=\sum_{i=i_{k-1}+1}^{3 n} a_{i}$, the term between the large brackets in (3) becomes $2 b_{k}^{2}-4 Q(n-k+1) b_{k}$, which is minimal if $b_{k}=(n-k+1) Q$.

If the 3-Partition instance has a yes-answer, implying that a perfect partition exists, then we can construct a tour $\widetilde{T}$ for which $\widetilde{b}_{k}=(n-k+1) Q$ for all $k \in\{2, \ldots, n\}$ (with $\widetilde{b}_{k}$ defined as $b_{k}$ with respect to $\widetilde{T}$ ), which inserted in (3) yields:

$$
\begin{aligned}
C^{O P T} & =C+R+\sum_{k=2}^{n}\left(2 b_{k}^{2}-4 Q(n-k+1) b_{k}\right) \\
& \leq C+R+\sum_{k=2}^{n}\left(2 \widetilde{b}_{k}^{2}-4 Q(n-k+1) \widetilde{b}_{k}\right) \\
& =C+R-2 Q^{2} \sum_{k=2}^{n}(n-k+1)^{2} \\
& =C+R-\frac{1}{3} Q^{2} n(n-1)(2 n-1) \\
& <C+4 Q n^{4}-\frac{1}{3} Q^{2} n(n-1)(2 n-1) \\
& =C+2 K^{2}-\frac{1}{3} Q^{2} n(n-1)(2 n-1),
\end{aligned}
$$

using $Q=K P$ and $K=2 P n^{4}$ in the second term of the last line.

Notice that $b_{k}$ is a multiple of $K$ for any $k \in\{2, \ldots, n\}$. Therefore standard calculus tells us that

$$
\sum_{k=2}^{n} 2 b_{k}^{2}-4 Q(n-k+1) b_{k}<-\frac{1}{3} Q^{2} n(n-1)(2 n-1)+2 K^{2}
$$

if and only if

$$
b_{k}=(n-k+1) Q, \text { for all } k \in\{2, \ldots, n\} .
$$

Therefore if no perfect partition exists then

$$
\begin{aligned}
C^{O P T} & =C+R+\sum_{k=2}^{n}\left(2 b_{k}^{2}-4 Q(n-k+1) b_{k}\right) \\
& \geq C-\frac{1}{3} Q^{2} n(n-1)(2 n-1)+2 K^{2} .
\end{aligned}
$$

We conclude that the 3-Partition instance has a yes-answer if and only if

$$
C^{O P T}<C-\frac{1}{3} Q^{2} n(n-1)(2 n-1)+2 K^{2},
$$

which completes the proof.

In the remaining part of the proof we show that the numbers $m$ and $M$ can be bounded by a polynomial in the size of the 3 -Partition instance, and still satisfy 
the conditions that each edge $\left(r, v_{i 1}\right)$ is traversed exactly once in each direction, and that vertex $u_{i}$ is visited before vertex $z_{i}$.

Assume that the optimal tour traverses the edge $\left(r, v_{i 1}\right)$ more than once in each direction. For large enough $M$ we can adjust the optimal tour to obtain a tour with smaller total completion time as follows. When the optimal tour visits vertex $v_{i 1}$ for the first time we continue this tour by visiting all the remaining vertices of root branch $i$ in the same order as they are visited in the optimal tour. Next, we continue the optimal tour, leaving out the visits to root branch $i$ (except for vertex $r$ ). For any vertex, the delay in completion time in this new tour is at most $2\left((2 n-2+2 Q) a_{i}+m\right)$. On the other hand, for at least one of the vertices of root branch $i$, the completion time is decreased by at least $2 l_{i}$. Therefore, the total decrease in the total completion time is at least $2 l_{i}-2\left((2 n-2+2 Q) a_{i}+m\right) W$, where $W=\frac{3}{2} n^{2}(n-1)+3 n+2 n Q$ is the total weight of the tree. Now it easy to see that we can choose $M$ such that $l_{i}>\left((2 n-2+2 Q) a_{i}+m\right) W$ for all $i \in\{1, \ldots, 3 n\}$ and $M$ is bounded by a polynomial in the size of the 3 -Partition instance and in $m$.

Now assume that the optimal tour traverses the edges $\left(r, v_{i 1}\right)(i=1 \ldots 3 n)$ exactly once in each direction but vertex $z_{i}$ is visited before vertex $u_{i}$ for some $i \in$ $\{1, \ldots, 3 n\}$. If we change the optimal tour by traversing root branch $i$ according to a 1-tour, then the completion time changes only for the vertices in root branch $i$. The decrease in completion time for vertex $u_{i}$ is exactly $2\left(m+(n-1) a_{i}\right)$, and the increase for the vertices $v_{i 2}, \ldots, v_{i n}, z_{i}$ is exactly $4 Q a_{i}$. Therefore, the total decrease becomes $2\left(m+(n-1) a_{i}\right)-4 Q a_{i}\left(n(n-1) / 2+a_{i}\right)$ which is a positive number for $m>2 Q a_{i}\left(n(n-1) / 2+a_{i}\right)-(n-1) a_{i}$.

We can strengthen Theorem 1 a little. We can reduce the MLP with polynomially bounded lengths and weights to the MLP with all vertex-weights 1 and all edge-lengths either 0 or 1 .

Corollary 1. The MLP is NP-hard for trees where all edge-lengths are either 0 or 1 .

Proof. Let $I$ be an instance of the tree-MLP with vertices $v_{1}, \ldots, v_{n}$ and edges $e_{1}, \ldots, e_{m}$. Let $w_{i}(i \in\{1, \ldots, n\})$ and $l_{j}(j \in\{1, \ldots, m\})$ be the weight of vertex $v_{i}$ and the length of edge $l_{j}$ respectively. From $I$ we define an instance $I^{\prime}$ of the tree-MLP with all vertex weights 1 and edge-lengths 0 and 1 .

We define weight $w_{i}^{\prime}=K w_{i}$ on vertex $v_{i}, i=1, \ldots, n$, where $K$ is a large number that we choose appropriately later. As mentioned before, vertex-weights may be regarded as an equivalent number of points at distance zero from each other. For edge $e_{j}$ with length $l_{j}$ incident to vertices $u$ and $v$ we insert $l_{j}-1$ extra vertices $v_{j 1}, \ldots, v_{j, l_{j}-1}$ and the path $u, v_{j 1}, \ldots, v_{j, l_{j}-1}, v$ containing $l_{j}$ edges of length 1 . The inserted vertices receive weight 1 and are referred to as intermediate vertices. We do this for all edges of $I$.

Choose $K>2 L(n+L-m)^{2}$, with $L$ the sum of the lengths of all edges of $I$. We claim that, for any positive integer $C$, there exists a solution with total 
completion time $C(I)<C$ for $I$ if and only if there exists a solution with total completion time $C\left(I^{\prime}\right)<K C$.

If there exists a tour $T^{\prime}$ for $I^{\prime}$ with $C\left(I^{\prime}\right)<K C$, then the tour for $I$ that follows from $T^{\prime}$ in the obvious way has total completion time less than $C$. On the other hand, if there exists a tour $T$ for $I$ with $C(I)=C-1$, then the tour $T^{\prime}$ for $I^{\prime}$ that follows from $T$ in the obvious way has objective $K(C-1)+D$, where $D$ is the total completion time of the intermediate vertices. It suffices to show that $D<K$.

The number of vertices of $I^{\prime}$ is $n+L-m$. Notice that $2 L(n+L-m)$ is an upper bound on the length of the tour that returns to the origin every time a new vertex has been visited, which in its turn is clearly an upper bound on the length of $T^{\prime}$. Therefore, $D \leq 2 L(n+L-m)^{2}<K$.

The following statement is an obvious corollary of the above results. It makes a clear distinction between edge-weighted and vertex-weighted trees.

Corollary 2. The MLP is strongly NP-hard for

(i) vertex-weighted trees, i.e. all edges have length 1,

(ii) edge-weighted trees, where all vertices have weight 1 and the edge-lengths have integer values larger than or equal to 1.

Proof. The first follows directly from Corollary 1 and the observation that we can replace any vertex of weight $w$ by $w$ vertices at distance zero from one another. The second follows from Corollary 1 as well. Given an instance $I$ of the tree-MLP with all edge-lengths either 0 or 1 (and all vertex-weights 1 by the definition of the tree-MLP), we define an instance $I^{\prime}$ of the tree-MLP by replacing an edge of length 0 by an edge of length 1 , and replacing an edge of length 1 by an edge of length $K$, where $K$ is some large number. It is easy to

verify that if we choose $K \geq 2 n^{2}$, then a tour is optimal for $I$ if and only if it is optimal for $I^{\prime}$.

\section{References}

1. F. Afrati, S. Cosmadakis, C. Papadimitriou, G. Papageorgiou, N. Papakostantinou, The complexity of the travelling repairman problem, RAIRO Informatique Theorique et Applications 20 (1986), 79-87.

2. S. Arora, G. Karakostas, Approximation schemes for minimum latency problems, Proceedings of the 31st ACM Symposium on Theory of Computing (Atlanta, 1999), 688-693.

3. S. Arora, G. Karakostas, A $2+\epsilon$ approximation for the $k$-MST problem, Proceedings of the 11th SIAM Symposium on Discrete Algorithms (SODA) (San Francisco, 2000), 754-759.

4. G. Ausiello, S. Leonardi, A. Marchetti-Spaccamela, On salesmen, repairmen, spiders, and other traveling agents, Proceedings of the 4 th Italian Conference on Algorithms and Complexity (Rome, Italy, 2000), Lecture Notes in Computer Science 1767, Springer, Berlin (2000), 1-16. 
5. I. Averbakh, O. Berman, Sales-delivery man problems on treelike networks, Networks 25 (1995), 45-58.

6. A. Blum, P. Chalasani, D. Coppersmith, W. Pulleyblank, P. Raghavan, M. Sudan, The minimum latency problem, Proceedings of the 26th ACM Symposium on the Theory of Computing (Montreal, Quebec, Canada, 1994), 163-171.

7. F.A. Chudak, T. Roughgarden, D.P. Williamson, Approximate $k$-MSTs and $k-$ Steiner trees via the primal-dual method and Lagrangean relaxation, Proceedings of the 8th International Conference on Integer Programming and Combinatorial Optimization (IPCO) (Utrecht, The Netherlands, 2001), Lecture Notes in Computer Science 2081, Springer, Berlin (2001), 60-70.

8. A. García, P. Jodrá, J. Tejel, A note on the travelling repairman problem, Pre-publications del seminario mathematico 3, University of Zaragoza, Spain (2001).

9. M.R. Garey, D.S. Johnson, Complexity results for multiprocessor scheduling under resource constraints, SIAM Journal of Computing 4 (1975), 397-411.

10. N. Garg, A 3-approximation for the minimum tree spanning $k$ vertices, Proceedings of the 37th Annual IEEE Symposium on Foundations of Computer Science (Burlington, VT, USA, 1996), IEEE Computer Society (1996), 302-309.

11. M. Goemans, J. Kleinberg, An improved approximation ratio for the minimum latency problem, Mathematical Programming 82 (1998), 111-124.

12. E. Koutsoupias, C. Papadimitriou, M. Yannakakis, Searching a fixed graph, Proceedings of the 23rd International Colloquium on Automata, Languages, and Programming (Paderborn, Germany, 1996), Lecture Notes in Computer Science 1099, Springer (1996), 280-289.

13. J.K. Lenstra, W.E. de Paepe, J. Sgall, R.A. Sitters, L. Stougie, Computer-aided complexity classification of dial-a-ride problems, to be published.

14. E. Minieka, The delivery man problem on a tree network, paper presented at the 1984 ORSA/TIMS San Francisco Meeting (1984).

15. E. Minieka, The delivery man problem on a tree network, Annals of Operations Research 18 (1989), 261-266.

16. S. Sahni, T. Gonzalez, P-complete approximation problems, Journal of the Association for Computing Machinery 23 (1976), 555-565.

17. D. Simchi-Levi, O. Berman, Minimizing the total flow time of $n$ jobs on a network, IIE Transactions 23 (1991), 236-244.

18. J.N. Tsitsiklis, Special cases of traveling salesman and repairman problems with time windows, Networks 22 (1992), 263-282.

19. I.R. Webb, Depth-first solutions for the deliveryman problem on tree-like networks: an evaluations using a permutation model, Transportation Science 30 (1996), 134147.

20. B.Y. Wu, Polynomial time algorithms for some minimum latency problems, Information Processing Letters 75 (2000), 225-229. 